\title{
The solid-state chemistry of rhodium(I) pincer complexes under extreme conditions
}

\author{
A. Longcake, J. P. Tidey, M. S. Senn* and A. B. Chaplin* \\ Department of Chemistry, University of Warwick, Gibbet Hill, Coventry, CV4 7AL \\ Alex.longcake@warwick.ac.uk
}

The activation of B-H bonds in $\sigma$-borane complexes is of interest not only due to their applications in catalysis (hydroborations, borylations), but because of the ambiguity of $\sigma$-borane coordination modes, which can be challenging to formerly define. ${ }^{[1]}$ Because $\sigma$ borane complexes are intermediates to B-H activation, they can be difficult to study due to the transience of the highly reactive species.

The $\sigma$-borane complexes $\left[\mathrm{Rh}(\mathrm{PONOP})\left(\eta^{2}-\mathrm{HBR}\right)\right]\left[\mathrm{BAr}_{4}^{\mathrm{F}}\right],\left(\mathrm{PONOP}=2,6-\mathrm{Bis}(\right.$ di-tert-butyl-phosphinito $)$ pyridine; Ar ${ }^{\mathrm{F}}=3,5-$ Bis(trifluoromethyl)phenyl; HBR = HBcat: 1; HBR = HBpin: 2) have been synthesised in good yields and have been established to undergo oxidative addition $(\mathrm{OA})$ in solution at modest temperatures $\left(<75^{\circ} \mathrm{C}\right)$. However, the OA products were unstable in solution, preventing their full characterisation using traditional solution-based methods. A single crystal high-pressure study of 1 was undertaken up to pressures of 25.8 kbar. An isomorphous phase transition was observed between the pressures of 4.8 and 8.8 kbar, which was accompanied by several geometrical rearrangements of the HBcat ligand with respect to the remainder of coordination complex (Fig. 1a). Most notably, the decrease in the N-Rh-B bond angle of $c a .6^{\circ}$ across the phase transition suggests an increased overlap between the metal $d$ orbital and the 'empty' boron orbital, indicating a stronger interaction between the metal centre and the $\sigma$-borane ligand (Fig. $1 \mathrm{~b} \& \mathrm{c}){ }^{[2]}$

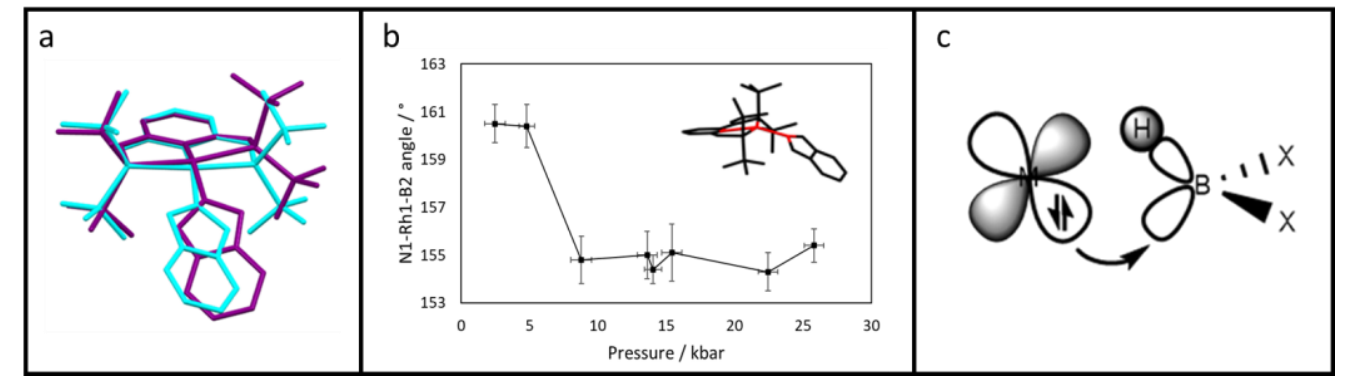

Figure 1. Pt. a) Superimposed structures of the cations in 1 before (cyan, 4.8 kbar) and after (purple, 8.8 kbar) the phase transition. Pt. b) Graph of the calculated N-Rh-B angle of $\mathbf{1}$ as a function of pressure; inset shows the angle of interest highlighted in red. Pt. $c$ ) Dewar-Chatt-Duncanson model of the back donation observed in $\sigma$-borane complexes, $\mathrm{X}=\mathrm{C}, \mathrm{O}$.

We aim to further the understanding of B-H bond activation by studying $\sigma$-borane complexes using high-pressure X-ray diffraction (HP$\mathrm{XRD)}$ as the principal analytical tool. Precise structural determination and analysis of a systematic series of $\sigma$-borane complexes will ultimately allow for better modelling of their reactive transition states in associated catalytic cycles, ultimately enabling better targeted design of industrially relevant catalysts.

[1] Hebden, T. J.; Denney, M. C.; Pons, V.; Piccoli, P. M. B.; Koetzle, T. F.; Schultz, A. J.; Kaminsky, W.; Goldberg, K. I.; Heinekey, D. M. (2008) J. Am. Chem. Soc. 130, 10812-10820.

[2] Marder, T. B.; Lin, Z., Contemporary Metal Boron Chemistry I. Springer-Verlag: Berlin Heidelberg, 2008; Vol. 130, p $125-127$.

Keywords: organometallic chemistry; B-H bond activation; pincer complexes; high-pressure crystallography

Alex Longcake acknowledges the Royal Society for a PhD studentship (RGF|EA 180160) and Diamond Light Source for time on Beamline I19 under proposal CY26847. 